\title{
Deep brain stimulation in Parkinson's disease: analysis of the variation in final stimulating lead placement based on multi-tract electrode recordings
}

\author{
Ryan Kurt Jacobs, ${ }^{1}$ Roger Melvill ${ }^{2}$ \\ ${ }^{1}$ Medical intern, Groote Schuur Hospital \\ ${ }^{2}$ Private neurosurgeon, Constantiaberg Medi-clinic
}

Corresponding author: Ryan Kurt Jacobs (ryankjacobs16@gmail.com)

\begin{abstract}
Background: Deep brain stimulation (DBS) of the subthalamic nucleus (SNT) is a treatment modality for Parkinson's Disease (PD). Either single central trajectory tract or multiple selected trajectory tracts based on microelectrode recordings (MER) are used for the placement of the final stimulating electrodes. This study aims to explore how many times trajectory tracts, other than the central tract are used for final lead placement.

Methods: Retrospective analysis of a randomly selected convenience sample of 24 subjects from patients who had DBS by a single neurosurgeon. After MRI and CT assessment, planning using a stereotactic frame for variable trajectory placement of temporary electrodes and MER that was the basis for site and tract selection for the final electrode placements used for DBS.

Results: Twenty four patients had 47 DBS electrodes placed: 1 unilateral and 23 bilateral. The central tract was used in 45 $(95.75 \%)$ of these cases. The central trajectory tract accounted for $30(63.83 \%)$, the anterior trajectory tract for $7(14.89 \%)$, the medial tract for $5(10.64 \%)$, the posterior for $4(8.51 \%)$ and the lateral for $1(2.13 \%)$ of final lead placements.

Conclusion: The results of this study based on the predicted best stimulating sites following MER show that alternates to the central trajectory tract are required in $37 \%$ of site placements. A comparative study exploring clinical benefit is required to assess if variable electrode trajectory placement based on accurate physiological measurements is superior to single central trajectory placement.
\end{abstract}

Keywords: DBS, Parkinson's disease, STN, final lead position

S Afr J Surg 2018;56(4)

http://dx.doi.org/10.17159/2078-5151/2018/v56n4a2566

\section{Introduction}

Deep brain stimulation (DBS) of the subthalamic nucleus (SNT) as treatment for advanced Parkinson's disease has become one of the best and more favoured management modalities. The optimal placement method of the stimulating electrodes is however contested. A review on DBS for Parkinson's disease suggests that MER does not significantly influence the outcome of the procedure, and another study reported that MER only changed the target locations by approximately $1 \mathrm{~mm}$ once again with little or no beneficial clinical outcome. ${ }^{1,2}$ On the other hand, the use of intraoperative MER has become more popular across the world, especially in determining the best position for the placement of the final stimulating electrode. ${ }^{4}$ Further controversy exists with regards to the efficacy of single and multiple intraoperative MER tracts in determining the best position for the final electrode. It is generally believed that sufficient STN activity can be detected with a single central trajectory tract with precise trajectory planning and interpretation of MER. ${ }^{3}$ Multiple simultaneously implanted microelectrodes however provide more accurate mapping the electrophysiological boundaries of the STN leading to a more accurate implantation position for the final stimulating electrode. ${ }^{4}$ This study aimed to explore how many times trajectory tracts, other than the central trajectory tract (which is widely used as the default single trajectory tract) are used for final lead placement.

\section{Subjects and methods}

Twenty-four study subjects who had been assessed and underwent DBS of the STN for PD by a single neurosurgeon were randomly selected by convenience sample. They had been assessed and the diagnosis of PD confirmed by a neurologist. Each patient was clinically (physically and psychologically) assessed and according to the Movement Disorder Society-Sponsored Unified Parkinson's Disease Rating Scale (MDS-UPDRS), to determine eligibility for surgery. The MDS-UPDRS is a diseases specific measurement 
tool comprised of a 30-minute questionnaire and an objective assessment completed by an interviewer. ${ }^{5}$ It consists of four parts each with a severity rating-scale ranging from 0 to 4 ; with $0=$ normal, $1=$ slight, $2=$ mild, $3=$ moderate, $4=$ severe. $^{6}$ Part 1 assesses the patient's "non-motor experiences of daily living" (i.e. behaviour and mood), Part 2 focuses on the "motor experiences of daily living" (i.e. frequency and severity of experienced symptoms, namely; bradykinesia, 'freezing', tremor, etc.). Part 4 aims to explore the nature (and frequency) of "motor complications" (i.e. dyskinesia, dystonia and motor fluctuations) which are as a result of medication use. Parts 1,2 and 4 are all subjective experiences of either the patient and/or the care-giver. Part 3 is the only part of the measurement tool which is objectively assessed by the interviewer, and it is based on the findings of the "motor examination". No specific (cut-off) score is used to determine a patient's eligibility for DBS, but rather the overall/ holistic picture is taken into account when deciding on eligibility for surgery. Data collection of the 24 patients medical records was done in retrospect, during October 2015. The data was qualitatively analysed using frequency tables.

\section{Procedure overview}

Meticulous preoperative planning is imperative to ensure optimal results. MRI imaging is used to locate the deep structures within the brain. On the morning of or the day before surgery, a stereotactic CT brain scan is done. Once both imaging modalities are acquired, the neurosurgeon, and neuroscientists, uses a computer generated program to merge the MRI and CT images for stereotaxis, to plan the safest route to the STN, while avoiding major blood vessels and central nervous system tissue tracts. The program (designed by medical technology company Medtronic $\AA$ ) simultaneously determines the ideal number of electrodes, and their subsequent specific configuration. It is important to note that the standard number of trajectory tract is in the planning phase is 5, but owing to patient to patient variability in anatomical structures within the brain, the final number of trajectory tracts that can be used intraoperatively, as well as the specific configuration thereof, is variable. The number of trajectories used can range from 1 to 5 per affected side. Once the neurosurgeon and neuroscientist are happy with the planned trajectory tracts and configuration, surgery is then able to commence. The final trajectory tracts are selected on the basis of meticulous MER as well as objective clinical response following stimulation from each of the implanted electrodes in the said configuration (macro-stimulation). The MER clearly indicates which of the tracts show the best STN activity, which in turn allowed for subsequent awake macrostimulation of the region. These two methods, namely MER in conjunction with awake macro-stimulation serve to determine the precise area of placement of the final stimulating electrode in the area where the maximal beneficial effects and least adverse effects are experienced. After the final stimulating lead has been placed, it is connected to a subcutaneously implanted device/pacer on the patient's chest.

\section{Results}

\section{Study demographics}

The study comprised 24 patients between the ages of 30 and 75 years. The duration of Parkinson's disease was determined by evaluating patient records. Both the age at which surgery was performed and nature (i.e. unilateral vs. bilateral) of surgery was also established. Table 1 describes the characteristics of the recruited subjects. The main complaints of participants were documented as: tremors, bradykinesia, rigidity, poor dexterity or medication associated dyskinesia. Table 2 describes the frequency of the main complaint or symptom as experienced by patients.

\begin{tabular}{lc} 
Table 1. Patient Characteristics of the 24 patients \\
\hline Sex \\
Male & 18 \\
Female & 6 \\
Duration of disease (in years) & \\
Mean & 8.65 \\
Range & $2-18$ \\
Age at surgery (in years) & \\
Mean & 57.8 \\
Range & $30-75$ \\
Nature of surgery & \\
Unilateral STN stimulation & 1 \\
Bilateral STN stimulation & 23
\end{tabular}

Table 2. Main complaints

\begin{tabular}{lc}
\hline Main complaint & Frequency \\
\hline Tremor & 9 \\
Bradykinesia & 8 \\
Dyskinesia & 5 \\
Poor dexterity & 1 \\
Rigidity & 1
\end{tabular}

\section{Results}

Specific microelectrode trajectory tract configurations were used for each patient (minimum 1, maximum 5) depending on the merged MRI and stereotactic CT scans trajectory planning route. Table 3 demonstrates the number of trajectories used in the various (predetermined) configurations. Each of the predetermined configurations was used for testing and determining the best position for the placement of the final stimulating lead, as described above. Although it is evident that the central tract is used in $45(n=47)(95.75 \%)$ of these patients, it was found that the final lead position trajectory in $30(63.83 \%)$ positioning's was the central tract, the anterior trajectory tract in $7(14.89 \%)$, the medial trajectory tract in $5(10.64 \%)$, the posterior in $4(8.51 \%)$ and the lateral in 1 $(2.13 \%)$. 


\begin{tabular}{lc}
$\begin{array}{l}\text { Table 3. Number of trajectories and specific configurations } \\
\text { used in 24 patients }\end{array}$ \\
\hline $\begin{array}{l}\text { Number of trajectories used and specific } \\
\text { configuration }\end{array}$ & $\begin{array}{c}\text { Number of } \\
\text { times used }\end{array}$ \\
\hline $\mathbf{1}$ & \\
C & 1 \\
A & 1 \\
$\mathbf{2}$ & \\
C; A & 5 \\
C; P & 1 \\
3 & \\
C; P; L & 1 \\
C; A; M & 2 \\
C; A; P & 3 \\
C; P; M & 1 \\
C; A; L & 2 \\
4 & \\
C; A; M; P & \\
C; A; L; P & 9 \\
A; M; L; P & 4 \\
5 & 1 \\
C; A; L; P; M & \\
\hline & \\
\hline &
\end{tabular}

Key: C - Central; A - Anterior; L - Lateral; P - Posterior; $\mathrm{M}-$ Medial

Table 4. Shows that significant variation in the final trajectory tracts between right-sided and left-sided leads exists.

\begin{tabular}{lccc}
\multicolumn{4}{l}{ Table 4. Trajectory of final lead } \\
\hline Position & $\begin{array}{c}\text { Right side (\%) } \\
\mathbf{n = 2 3}\end{array}$ & $\begin{array}{c}\text { Left side (\%) } \\
\mathbf{n = 2 4}\end{array}$ & $\begin{array}{c}\text { Total (\%) } \\
\mathbf{n = 4 7}\end{array}$ \\
\hline Central & $18(78.26 \%)$ & $12(50 \%)$ & $30(63.83 \%)$ \\
Anterior & $4(17.39 \%)$ & $3(12.5 \%)$ & $7(14.89 \%)$ \\
Lateral & - & $1(4.17 \%)$ & $1(2.13 \%)$ \\
Posterior & $1(4.35 \%)$ & $3(12.5 \%)$ & $4(8.51 \%)$ \\
Medial & - & $5(20.83 \%)$ & $5(10.64 \%)$
\end{tabular}

\section{Discussion}

This study shows, contrary to current practice and belief, that the central trajectory tract is not always the sole and best option for placement of the final stimulating lead. The central tract is one of the most commonly used tracts in the multi-tract system for testing and stimulating the STN during DBS. However, on occasion, the central tract is used as the sole trajectory tract in cases where STN target location is planned and verified using MRI imaging alone (in the absence of MER and/ or awake macro-stimulation). ${ }^{7}$ Although evidence supports this method of STN-DBS in improving the motor symptoms of those with PD, it is noted that on rare occasions (owing to anatomical variations in brain structure), the use of the central tract may lead to detrimental effects, thus necessitating its omission when performing this procedure. ${ }^{8,9}$ In this present study sample, it was found that the central tract is only used as the final lead placement in $63.83 \%$ of reported cases, which is significantly less than previously reported literature. This therefore emphasises the importance of accurate preoperative and intraoperative planning and microelectrode testing, in conjunction with awake macro-stimulation, to assess real-time clinical response. At some centres where DBS is performed, it is believed that MER is unnecessary and increases the risk of intracranial haemorrhage, and that the targeting of the STN is sufficient using MRI scans. ${ }^{9-12}$ However, Benazzouz A et al. ${ }^{4}$ in their study have concluded that "microelectrode recording improves the accuracy of the electrode implantation into the subthalamic nucleus." Umemura A et al. ${ }^{3}$ have adopted a more selective strategy for electrode placement. They postulated that sufficient STN activity can be obtained with minimal MER trajectories often with a single central tract, and that should none-beneficial results occur with this single central tract, that an anterior or posterior rather than medial or lateral approach should be used for the placement of the final stimulating lead. However, as many as possible (maximum 5) trajectory tracts provide more information about the electrophysiological boundaries of the STN, and consequently lead to better determining of the best position for the final stimulating lead, while using MER. ${ }^{4}$ As illustrated in this study, various trajectory tracts were used in 45 (95.75\%) of cases, with no serious adverse effects occurring. The use of the multiple tracts aided in the positioning of the final lead, showing that anterior (14.89\%) and medial (10.64\%) positions were more favoured as final lead placement tracts after the central tract $(63.83 \%)$. It has also been previously demonstrated that a larger number of MER lead to better motor outcomes following DBS for Parkinson's disease. ${ }^{13}$ Improved motor outcomes are ultimately the goal of STN stimulation for Parkinson's disease.

This small scale study sample of 24 patients shows that the central tract might account for $63.83 \%$ of final lead placements. This number is significantly less than previously thought and expected. Furthermore, from the results displayed in Table 4, it is noted that a significant variation in the final trajectory tracts between right-sided and left-sided leads exists. This may be attributed to the unique variability in individual anatomical structures of the brain or following significant brain shift following the implantation of the final lead in the contra-lateral side, ${ }^{3}$ thus further emphasising the importance of the use of intraoperative MER as well as (awake) macro-stimulation in determining the best, reliable position of the final stimulating lead.

\section{Conclusion}

It is largely assumed that the single central trajectory tract, without intraoperative MER and awake macro-stimulation, can be used for final lead placement in STN DBS. ${ }^{12,13}$

The use of merged preoperative MRI and stereotactic CT scans in target planning in conjunction with intraoperative MER and awake macro-stimulation in determining the 
position of the final stimulating lead, shows that the central trajectory tract cannot be routinely used as the default tract as in $37 \%$ of patients an alternate trajectory tract had to be used. This suggests that these stereotactic guided adjuncts optimise the lead placement. However, for clinical benefit of variable electrode trajectory placement based on accurate physiological measurements over single (anatomically guided) central trajectory placement to be proven, a well-designed prospective analysis of the two techniques is required.

\section{REFERENCES}

1. Boucai L, Cerquetti D, Merello M. Functional surgery for Parkinson's disease treatment: A structured analysis of a decade of published literature. Br J Neurosurg. 2004;18(3):213-22. doi: $10.1080 / 02688690410001732625$

2. Senatus PB, Teeple D, McClelland S, Pullman SL, Yu Q, Ford $\mathrm{B}$, et al. A technique for minimally altering anatomically based subthalamic electrode targeting by microelectrode recording. Neurosurg Focus. 2006;20(5):1-4. doi: 10.3171/foc.2006.20.5.9

3. Umemura A, Oka Y, Yamada K, Oyama G, Shimo Y, Hattori $\mathrm{N}$. Validity of single tract microelectrode recording in subthalamic nucleus stimulation. Neurol Med Chir. 2013 November;53:821-7. doi: 10.2176/nmc.oa2012-0412

4. Benazzouz A, Breit S, Koudsie B, Pollak P, Krack P, Benabid AL. Intraoperative Microrecordings of the Subthalamic Nucleus in Parkinson's Disease. J Mov Disord. 2002;17(3):145-9. doi: 10.1002/mds. 10156

5. Goetz CG, Fahn S, Martinez-Martin P, et al. Movement disorder society-sponsored revision of the Unified Parkinson's disease rating scale (MDS-UPDRS): process, format, and clinimetric testing plan. J Mov Disord. 2017;22:41-7. doi: 10.1002/ mds. 22340

6. Goetz CG, Tilley BC, Shaftman SR, Stebbins GT, Fahn S, Martinez-Martin P, et al. Movement Disorder SocietySponsored revision of the unified Parkinson's Disease Rating
Scale (MDS-UPDRS): Scale Presentation and Clinimetric Testing Results. J Mov Disord. 2008;23(15):2129-70. doi: 10.1002/mds. 21198

7. Holl EM, Petersen EA, Foltynie T, Martinez-Torres I, Limousin P, Hariz MI, Zrinzo L. Improving targeting in imageguided frame-based deep brain stimulation. Neurosurgery. 2010;67:437-47. doi: 10.1227/NEU.0b013e3181f7422a

8. Al-Helli O, Thomas DL, Massey L, Foltynie T, Limousin P, Hotlon JL, et al. Deep brain stimulation of the subthalamic nucleus: histological verification and 9.4-T MRI correlation. Acta Neurochir, 2015;157(12):2143-7. doi: 10.1007/s00701015-2599-x

9. Foltynie T, Zrinzo L, Martinez-Torres I, Tripoliti E, Petersen E, Holl E, et al. MRI-guided STN DBS in Parkinson's disease without microelectrode recording: efficacy and safety. J Neurol, Neurosurg Psychiatry. 2011;82(4):358-63. doi: 10.1136/ jnnp.2010.222497

10. Andrade-Souza YM. Schwalb JM, Hamani C, Eltahawy H, Hoque T, Saint-Cyr J, et al. Comparison of three methods of targeting the subthalamic nucleus for chronic stimulation in Parkinson's disease. Neurosurgery. 2008;62(2):875-83. DOIdoi: 10.1227/01.NEU.0000156547.24603.EE

11. Benjjani BP, Dormont D, Pidoux B, Yelnik J, Damier P, Arnulf I, et al. Bilateral subthalamic stimulation for Parkinson's disease by using three-dimensional stereotactic magnetic resonance imaging and electrophysiological guidance. Neurosurgery. 2000;92:615-25. doi: 10.3171/jns.2000.92.4.0615

12. Hariz M. Safety and risk of microelectrode recording in surgery for movement disorders. Stereotact Funct Neurosurg. 2002;78(3-4):146-57. doi: 10.1159/000068960

13. Temel Y, Wilbrink P, Duits A, Boon P, Tromp S, Ackermans L, et al. Single electrode and multiple electrode guided electrical stimulation of the subthalamic nucleus in advanced Parkinson's disease. Neurosurgery. 2007;61(2):346-55. doi: 10.1227/01. neu.0000303993.82149.98 